\title{
The Effect of Survivin on Multidrug Resistance Mediated by P-Glycoprotein in MCF-7 and Its Adriamycin Resistant Cells
}

\author{
Feng Liu, ${ }^{a, b}$ Zhen-Hua XIE, ${ }^{a}$ Guo-Ping CAI, ${ }^{a}$ and Yu-Yang JiAnG ${ }^{*, a, c}$ \\ ${ }^{a}$ The Key Laboratory of Chemical Biology, Guangdong Province, Division of Life Science, Graduate School at Shenzhen, \\ Tsinghua University; Shenzhen 518055, China: and ${ }^{b}$ Department of Biological Sciences and Biotechnology, Tsinghua \\ University; and ${ }^{c}$ Key Laboratory of Bioorganic Phosphorus Chemistry and Chemical Biology, Ministry of Education, \\ Department of Chemistry, Tsinghua University; Beijing 100084, China. \\ Received May 29, 2007; accepted August 30, 2007
}

\begin{abstract}
Although anticancer chemotherapeutic drugs have been designed to inhibit the growth of tumor cells, chemotherapy frequently fails due to the development of multidrug resistance (MDR). In this paper, the effect of survivin on multidrug resistance mediated by P-glycoprotein (Pgp) was investigated in breast cancer cells. Overexpression of survivin in MCF-7 cells transfected with survivin expression vector $\mathrm{pEGFP/survivin} \mathrm{results} \mathrm{in} \mathrm{de-}$ creasing sensitivity to anticancer drugs and activation of Pgp to export drug out of cells. Down regulation of survivin in MCF-7/adriamycin (ADR) transfected with RNAi directed against survivin vector psh1/survivin could increase the drug accumulation in cells by inhibiting Pgp. Downregulation of the expression of the Pgp with the specific inhibitor verapamil could markedly suppress the survivin mRNA expression, whereas the reverse impact was not observed. Survivin might modulate the turnover of Pgp or transport by Pgp in cells, which result in antiapoptosis and drug resistance. Our results suggest that survivin might play a key role in MDR in the presence of Pgp, and this might represent a novel strategy for modulating MDR in cancer cells.
\end{abstract}

Key words survivin; P-glycoprotein; multidrug resistance; MCF-7 cell

Chemotherapy plays an important role in the management of patients with breast carcinoma. However, the treatment response rate is low and cases of complete remission are rare due to the development of multidrug resistance (MDR). Multidrug resistance is the phenomenon of simultaneous resistance to many structurally unrelated cytotoxic agents, which is one of the most formidable challenges in the field of cancer therapy. ${ }^{1)}$ A common mechanism of MDR is the active export of drug from cells by the overexpression of P-glycoprotein $(\mathrm{Pgp})^{2)}$ and other ATP-binding cassette (ABC) transporters. In previous studies, Shabbits et al. ${ }^{3)}$ reported a role for Pgp in mediating resistance to apoptosis. Recently, resistance to chemotherapy has been associated with decreased apoptosis, which has been a good marker of the chemo-resistance of tumor cells.

Survivin, one of the regulators of apoptosis that may participate in cancers, has been a focus of research. ${ }^{4,5)}$ Survivin is a structurally unique inhibitor of apoptosis (IAP), substances which block apoptosis induced by a variety of nonrelated apoptosis triggers, including different anti-tumor agents. $^{6-8)}$ Although the exact mechanism by which IAP family proteins suppress apoptosis is unclear, survivin is known to directly or indirectly bind and inhibit the terminal effector cell death protease cascades, caspase 3 and 7, as well as inhibit the activation of caspase 9 , the initiator in the mitochondrial pathway for apoptosis. ${ }^{9-11)}$ Normally, survivin expression is present in fetal tissues, but no survivin expression was detected in a majority of terminally differentiated adult tissues. However, survivin is prominently expressed in most common human cancers. ${ }^{12)}$ Furthermore, it has been reported that survivin is involved in the resistance to chemotherapy, ${ }^{13,14)}$ and may serve as a radio- and chemo-resistance factor. ${ }^{15)}$ Studies have shown that survivin expression was significantly higher after treatment with anti-cancer drugs in various cancer cells. ${ }^{16,17)}$ Thus, survivin might potentially play a key role in resistance to anti-cancer drugs. However, there is no information on the role of survivin in MDR in the presence of Pgp in cancer cells.

In this paper, the changes in mRNA expression between survivin and Pgp as well as their effects on chemo-resistance in MCF-7 cancer cells and adriamycin (ADR)-resistant MCF-7/ADR cells were investigated. We found that survivin plays a role in MDR in the presence of Pgp. In the future, we hope to clarify the role of survivin in MDR in the presence of Pgp.

\section{MATERIALS AND METHODS}

Chemicals and Reagents The cytotoxic drugs adriamycin (ADR) and mitoxantrone (MIT) were purchased from Wanle (Main Luck Pharmaceuticals Inc., China). Culture medium RPMI 1640, Dulbecco's modified Eagle medium (DMEM) and fetal bovine serum (FBS) were obtained from Gibco (Life Technologies, U.S.A.). Penicillin and streptomycin were obtained from Amresco (U.S.A.). 3-(4,5-Dimethylthiazol-2-yl) 2,5-diphenyltetrazolium bromide (MTT) was purchased from Sigma (St. Louis, MO, U.S.A.).

Cell Culture MCF-7, a human breast adenocarcinoma cell line, was obtained from the American Type Culture Collection (ATCC, Manassas, VA, U.S.A.), and its ADR resistant cell line MCF-7/ADR, which could propagate in 0.2 $\mu \mathrm{g} / \mathrm{ml}$ ADR, was obtained from Nanjing Keygen Biotech. Co., Ltd. (China). Briefly, MCF-7/ADR cells were developed by step-wise selection as previously described. ${ }^{18)} \mathrm{MCF}-7$ cells were routinely maintained in DMEM medium and MCF-7/ADR cells were cultured in RPMI 1640. Both media were supplemented with $10 \%$ fetal bovine serum, 100 units $/ \mathrm{ml}$ penicillin and $100 \mu \mathrm{g} / \mathrm{ml}$ streptomycin. The cells were grown in a humidified atmosphere at $37^{\circ} \mathrm{C}$ in $5 \% \mathrm{CO}_{2}$.

Drug Treatment The sensitive cell line $\mathrm{MCF}-7$ and its ADR resistant cell line MCF-7/ADR were divided into a blank control group and ADR treatment group. After treating 
the cells with various doses of drugs for different times, the cells were collected to examine the expression of Pgp and survivin.

Plasmid Construction and Transfection The anti-survivin shRNA vector was constructed according to the principle of shRNA designation. The shRNA targeting survivin sequence (nucleotides 486-504) was synthesized (Invitrogen) and inserted into ApaI/Hind III sites of pCMV5 to generate the survivin silencing vector psh1/survivin. The DNA plasmid encoding survivin was generated by reverse transcription PCR using the survivin forward primer $\left(5^{\prime}\right.$-CTCGGATCCATCCATGGCAGCCAGCTG-3 $\left.3^{\prime}\right)$ and reverse primer $\left(5^{\prime}-\right.$ GCTGAATTCGCCACCATGGGTGCCCCGACGTTG-3'). The PCR product was inserted into (BamHI/EcoRI) sites of pEGFP-N3 in fusion with EGFP to generate the plasmid $\mathrm{pEGFP} /$ survivin. The precision of both of the constructs was confirmed by sequencing. Transfection of cells with mammalian expression constructs by LipofectAMINE 2000 (Invitrogen, U.S.A.) was carried out according to the methods provided by the manufacturer's instructions. In brief, cells were plated into 6 -well plates $\left(2 \times 10^{5}\right.$ cells/well $) 18 \mathrm{~h}$ before transfection. The cells were transfected with plasmid DNA and transfection reagent at a DNA : reagent ratio of $1: 2$, and then incubated at $37^{\circ} \mathrm{C}$ in a $\mathrm{CO}_{2}$ incubator for $18-48 \mathrm{~h}$ prior to testing for gene expression.

Cell Proliferation Assays The cells were plated in 96well plates at a density of $2 \times 10^{4}$ cells/well and allowed to grow overnight. The cells were then treated with ADR and MIT for $48 \mathrm{~h}$ in complete medium. After drug treatment, 10 $\mu \mathrm{lof} 5 \mathrm{mg} / \mathrm{ml}$ MTT in phosphate buffered saline (PBS) was added to all wells and the cells incubated for another $4 \mathrm{~h}$. The surviving cells converted MTT to formazan that generates a blue-purple color when dissolved in dimethyl sulfoxide. The intensity of formazan was measured at $490 \mathrm{~nm}$ using a DTX 880 Multimode Detector (Beckman Coulter, U.S.A.). The relative percentage of survival was calculated by dividing the absorbance of treated cells by that of the control in each experiment. The $\mathrm{IC}_{50}$ was determined as the concentration of drug that produced a $50 \%$ reduction of absorbance at 490 nm.

RNA Extraction and Semiquantitative Reverse Transcriptase Polymerase Chain Reaction (RT-PCR) Total RNA was isolated from proliferating cells using Trizol reagent (Invitrogen) as recommended by the supplier. One microgram of purified RNA was analyzed using reverse tran- scription-polymerase chain reaction (RT-PCR) with an ImProm $^{\mathrm{TM}}$ Reverse Transcription System (Promega, U.S.A.). Glutaraldehyde-3-phosphate dehydrogenase (GAPDH) was used as an internal control. The RT reaction was performed at $25^{\circ} \mathrm{C}$ for $5 \mathrm{~min}$, then $42^{\circ} \mathrm{C}$ for $60 \mathrm{~min}$. Following inactivation at $70^{\circ} \mathrm{C}$ for $15 \mathrm{~min}$, PCR amplification was performed in an iCycler Thermal Cycler (Bio-Rad, U.S.A.) under the following reaction conditions: $94^{\circ} \mathrm{C}$ for $3 \mathrm{~min}, 58^{\circ} \mathrm{C}(\mathrm{Pgp})$ or $62^{\circ} \mathrm{C}$ (surviving and GAPDH) for $30 \mathrm{~s}, 72^{\circ} \mathrm{C}$ for $1 \mathrm{~min}$, and a final extension at $72^{\circ} \mathrm{C}$ for $5 \mathrm{~min}$. The amplification used 26 cycles for survivin and Pgp, and 22 cycles for GAPDH. PCR products $(5 \mu \mathrm{l})$ were analyzed by electrophoresis on $1.2 \%$ $(\mathrm{w} / \mathrm{v})$ agarose gel and were visualized by ethidium bromide staining under ultraviolet light. The results of electrophoresis were analyzed by a Gel DOC ${ }^{\mathrm{TM}}$ XR Imaging Assay System (Bio-Rad, U.S.A.). Relative gene expression was defined as the ratio of target gene expression to GAPDH gene expression. The sequence of primers used in the RT-PCR was as follows: survivin: 5' -TGGCTGCCATGGATTGAG-3' (sense) and 5'-TCTGAGGAGGCACAGGTGTG-3' (antisense); Pgp: 5'-AAAGCGACTGAATGTTCAGTGG-3' (sense) and 5'-AATAGATGCCTTTCTGTGCCAG-3' (antisense); GAPDH: 5'-CAACGTGTCAGTGGTGGACCTG-3' (sense) and 5' - TTACTCCTTGGAGGCCATGTGG-3' (antisense).

Measurement of ADR Accumulation After MCF-7 and MCF-7/ADR cells were transfected with $\mathrm{pEGFP/survivin}$ and psh1/surviving, respectively, for $48 \mathrm{~h}$, control and treated cells were incubated with $25 \mu \mathrm{M}$ of ADR for $2 \mathrm{~h}$. At the end of incubation, cells were washed three times with PBS and observed under a fluorescence microscope with $400 \times$ magnification (Olympus IX51, Olympus Corporation, Japan). The excitation and emission wavelengths for ADR were 468 $\mathrm{nm}$ and $550 \mathrm{~nm}$, respectively. As excited ADR could produce fluorescence, the cells that accumulated ADR will exhibit visible red light under a fluorescence microscope.

\section{RESULTS}

Expression of Survivin and Pgp mRNA in MCF-7 and MCF-7/ADR Cells The expression levels of survivin and Pgp were detected by RT-PCR in MCF-7 and MCF-7/ADR cells. Compared with MCF-7 cells, both survivin and Pgp mRNA expression increased markedly in MCF-7/ADR cells. As shown in Fig. 1, the relative expression rates of survivin and Pgp mRNA in MCF-7/ADR cells were about 4.5 and
A

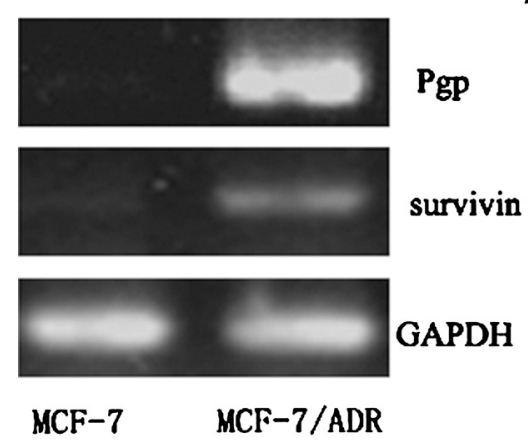

B

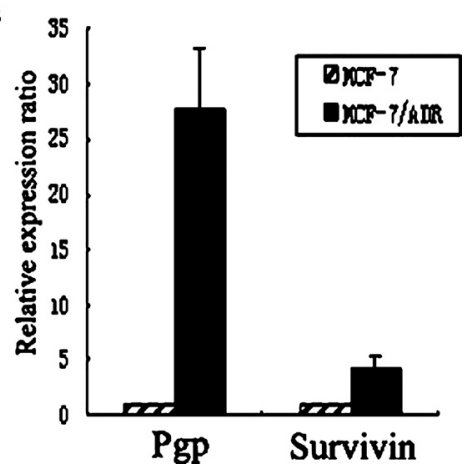

Fig. 1. Expression of Survivin and Pgp mRNA in MCF-7 and MCF-7/ADR Cells Using RT-PCR Analysis

Both survivin expression and Pgp mRNA expression in MCF-7/ADR cell were significantly higher than that in MCF-7 cells. The expression of mRNA was determined by densitometry scanning of the bands and then normalized to each parental MCF-7 cell expression band. Values are expressed as the mean \pm S.E.M. 
27.7 fold higher, respectively, than that of the MCF-7 cells.

Chemosensitizing Effects of Drugs in the MCF-7 Cells with or without pEGFP/Survivin Vectors In order to detect the chemoresistance changes in cells, the MTT assay was performed with MCF-7, MCF-7/ADR and MCF-7 transfected with survivin expressing vector $\mathrm{pEGFP} /$ survivin. As shown in Table 1, after exposure to ADR and MIT, the $\mathrm{IC}_{50}$ of MCF-7 cells was 0.88 and $0.81 \mu \mathrm{g} / \mathrm{ml}$, respectively. However, the $\mathrm{IC}_{50}$ of MCF-7/ADR cells was significantly higher than that of MCF-7 cells. The relative drug resistances of MIT and ADR in MCF-7/ADR cells were 205.6 and more than 113.6 greater than that of the MCF-7 cells. It is noteworthy that the survivin enhanced the resistance to both of the drugs in MCF-7 cells. The $\mathrm{IC}_{50}$ increased markedly in MCF-7 cells with $\mathrm{pEGFP/survivin} \mathrm{vector} \mathrm{when} \mathrm{it} \mathrm{was} \mathrm{treated}$ together with ADR. There was slight augmentation by MIT in MCF-7 cells with $\mathrm{pEGFP/survivin} \mathrm{vector.} \mathrm{These} \mathrm{results}$ indicate that the survivin was responsible for the increased drug resistance.

Survivin mRNA Expression Was Influenced by Pgp

Table 1. Chemosensitizing Effects of the ADR and MIT on Cells

\begin{tabular}{lcrrrr}
\hline \hline & \multicolumn{3}{c}{ ADR } & & \multicolumn{2}{c}{ MIT } \\
\cline { 2 - 3 } \cline { 5 - 6 } & \multicolumn{2}{c}{$\mathrm{IC}_{50}$} & $\mathrm{RF}$ & $\mathrm{IC}_{50}$ & $\mathrm{RF}$ \\
\hline MCF-7 & $0.88 \pm 0.18$ & & & $0.81 \pm 0.32$ \\
MCF-7+pEGFP & $1.12 \pm 0.22$ & & $0.98 \pm 0.17$ & \\
MCF-7/ADR & $>100$ & $>113.6$ & & $166.56 \pm 21.87$ & 205.6 \\
MCF-7+pG/sur & $72.4 \pm 1.53$ & 64.6 & & $2.28 \pm 0.72$ & 2.32 \\
\hline
\end{tabular}

$\mathrm{IC}_{50}$ values $(\mu \mathrm{g} / \mathrm{ml})$ were determined using the MTT assay. Cells $\left(2 \times 10^{4}\right)$ were seeded and exposed to ADR or MIT for $48 \mathrm{~h}$ with and without $\mathrm{pEGFP} /$ survivin vector. Vaules are means \pm S.D. of at least three independent experiments. pG/sur: pEGFP/survivin. RF: Relative resistance fold.

A.

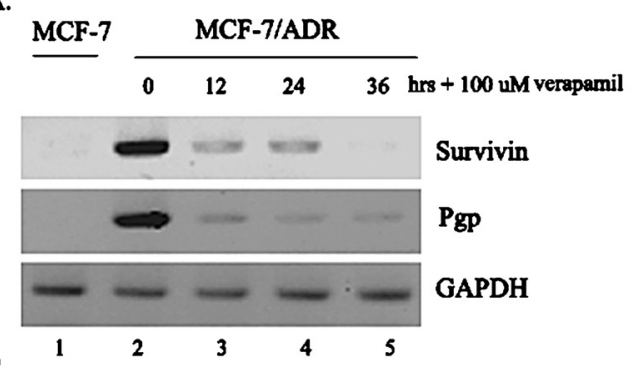

c.

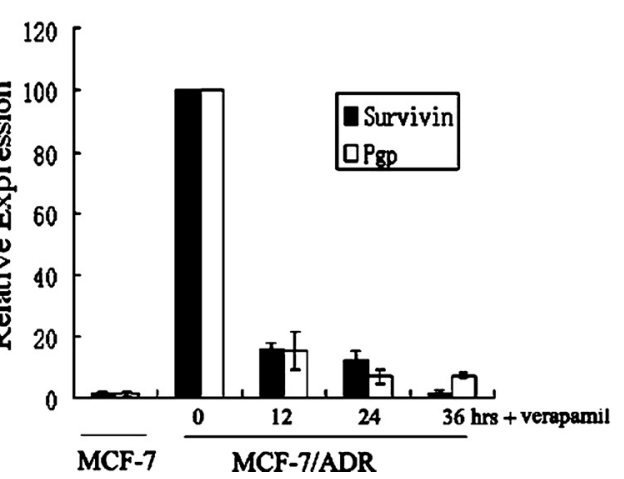

Level To study the changes in mRNA expression between Pgp and survivin, RT-PCR was performed using samples from control and treated cells. Along with the decreased expression of Pgp in MCF-7/ADR cells treated with the Pgp specific inhibitor verapamil, the mRNA expression level of survivin was also significantly suppressed (Figs. 2A, C). Approximately $90 \%$ of the survivin mRNA was blocked after incubation with verapamil for $12 \mathrm{~h}$. The survivin gene was undetectable after $36 \mathrm{~h}$ of verapamil treatment. Therefore, survivin RNAi was used. As shown in Figs. 2B and D, when survivin mRNA expression was retarded by its specific shRNA plasmid psh1/survivin, there was a slight decrease in the expression of Pgp, whereas a distinct change was found in MCF-7/ADR cells transfected with psh1/survivin for $72 \mathrm{~h}$.

Effect of Survivin on Pgp Function We also studied the effect of survivin on the accumulation of ADR, a Pgp transportable chemotherapeutic drug, in both cells. Compared with control, the intracellular accumulation of ADR increased in MCF-7/ADR cells transfected with the survivin targeting RNAi construct psh1/survivin (Figs. 3A-C). In addition, the accumulation of ADR decreased in cells expressing survivin gene fusion with GFP after transfection with $\mathrm{pEGFP} /$ survivin for $48 \mathrm{~h}$ in MCF-7 cells (arrowheads, Figs. 3E-F). The results described above indicate that survivin is responsible for the increased efflux of drug out of cells by Pgp.

\section{DISCUSSION}

The development of refractory disease in breast carcinoma is frequently associated with the progression of MDR. There are several mechanisms of MDR. The best characterized mechanism is membrane transport proteins, which are capa-

B.
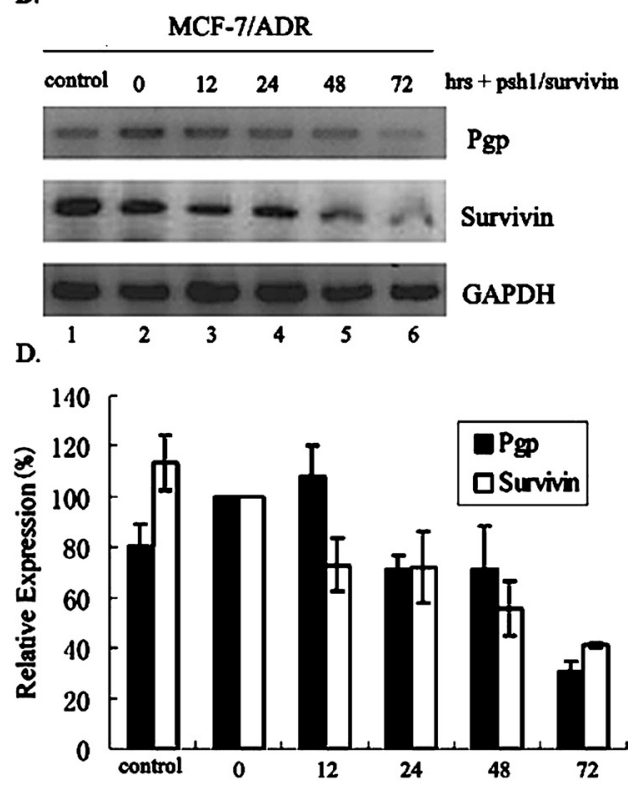

Fig. 2. Changes in Survivin and Pgp mRNA Expression in MCF-7/ADR Cells

(A, C) Survivin expression was detected in the presence of the Pgp inhibitor verapamil (100 $\mu \mathrm{M})$. After the cells were treated with verapamil for $12 \mathrm{~h}$, the expression of survivin was significantly decreased (Lane 3). Survivin was almost undetectable in the presence of verapamil for $36 \mathrm{~h}$ (Lane 5). (B, D) Pgp expression was estimated in the presence of survivin targeting RNAi plasmid psh1/survivin. The mRNA expression of Pgp was slightly influenced by the expression of survivin, which was suppressed by the psh1/survivin vector. The expression of mRNA was determined by densitometry scanning of the bands and then normalized to each cell without treatment with verapamil or pshi/survivin expression band. Values are expressed as the mean \pm S.E.M. 
A

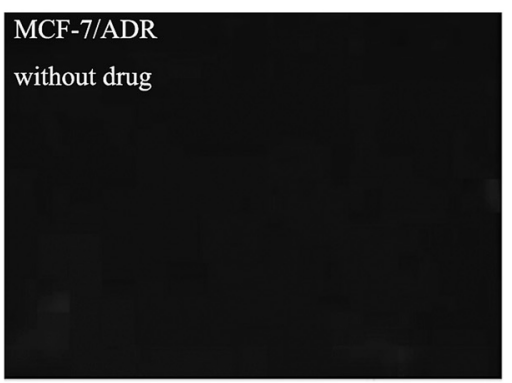

D

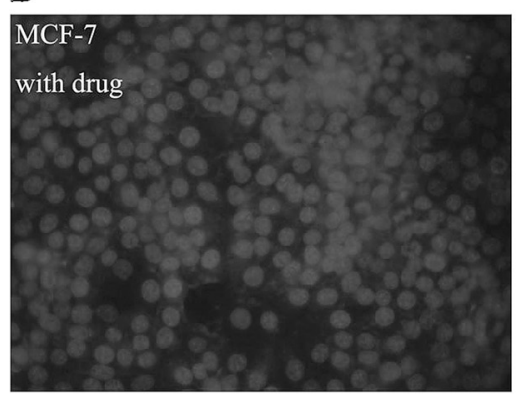

B

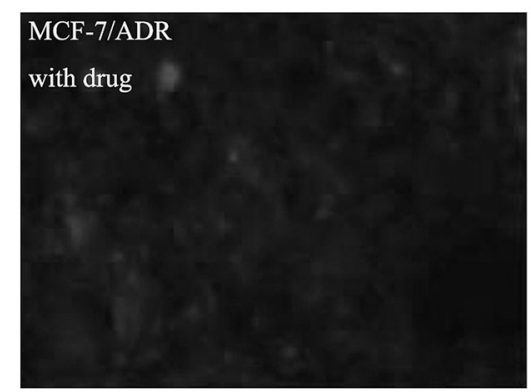

E

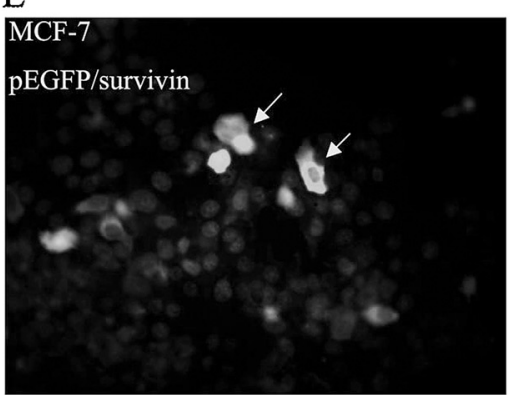

C

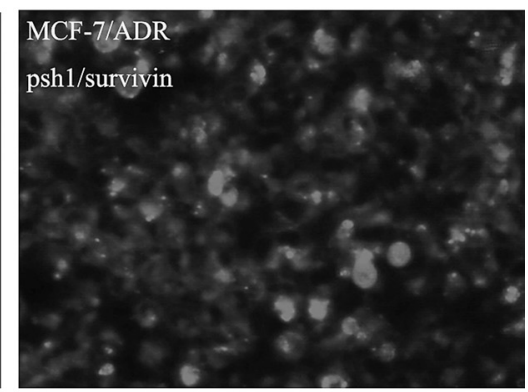

F

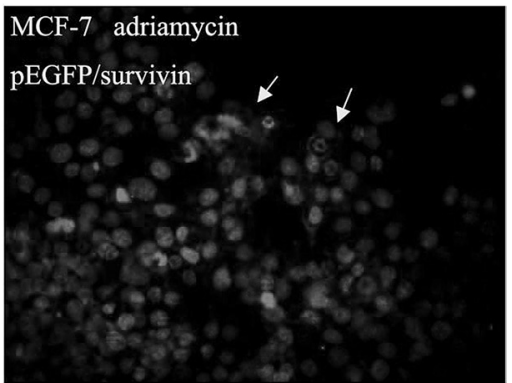

Fig. 3. Effect of Survivin on ADR Accumulation in MCF-7/ADR $(\mathrm{A}-\mathrm{C})$ and MCF-7 (D-F) Cells

MCF-7/ADR cells were transfected with psh1/survivin directly against survivin. MCF-7 cells were transfected with pEGFP/survivin. Cells were then incubated with $25 \mu \mathrm{M}$ ADR for $2 \mathrm{~h}$, followed by washing three times with PBS. ADR accumulation was observed under a fluorescence microscope with $400 \times$ lens. Enhanced ADR accumulation was observed after the survivin expression was interfered with psh1/survivin(C). In MCF-7 cells, expression of survivin with pEGFP/survivin increased the activity of the cells to pump ADR out of the cells (E, F arrowheads). Results are representative of three similar experiments.

ble of reducing the intracellular accumulation of drugs by excluding a drug outside the cell. ${ }^{19)}$ Pgp, one of the membrane transporters, can result in resistance to a wide range of naturally occurring products such as anthracyclines, vinca alkaloids, dactinomycin and taxanes. ${ }^{20)}$ In addition to the membrane transporter, apoptosis inhibition is also an important mechanism of drug resistance. As a member of the IAP family, survivin has received much more attention in recent years not only with respect to its anti-apoptosis effect, but also chemoresistance during cancer treatment.

Previous reports have revealed that survivin mRNA or protein expression is correlated with an unfavorable prognosis in patients with various malignant tumors. ${ }^{21,22)}$ Survivin is also involved in drug resistance in chemotherapy. ${ }^{13,14)}$ In the present study, we found survivin mRNA expression was much higher in MCF-7/ADR cells than in the parental sensitive MCF-7 cells (Fig. 1). Another result in our study demonstrated that the cell sensitization to ADR and MIT was distinctly decreased in MCF-7 cells transfected with $\mathrm{pEGFP/survivin} \mathrm{(Table} \mathrm{1).} \mathrm{This} \mathrm{is} \mathrm{in} \mathrm{agreement} \mathrm{with} \mathrm{other}$ evidence which shows a change in survivin expression contributes to chemotherapy resistance. ${ }^{23)}$ Taken together, a change in survivin expression may enhance the chemoresistance to various drugs and prolong the lifespan of cancer cells.

Consistent with another study, which indicated that HL60R cells expressed much more Pgp as well as survivin than HL60 cells, ${ }^{24)}$ we also detected significant overexpression of Pgp in MCF-7/ADR cells. Although the correlation between Pgp and survivin expression is still debated in drug resistance, the overexpression of Pgp together with the upregulation of survivin was determined in association with the poor prognosis of the patients. ${ }^{25)}$ In our report, the mRNA expression of survivin was significantly suppressed after the Pgp was inhibited by its specific inhibitor verapamil. Verapamil, a calcium channel blocker, inhibits the efflux function of Pgp and cytoskeletal transfer and therefore plays an important role in circumvention of native and acquired chemoresistance of cancer cells. It was demonstrated that verapamil could bind directly to Pgp or suppress the $m d r l$ gene (encoding Pgp) proximal promoter activity to inhibit Pgp. ${ }^{26,27)}$ However, there have been no reports indicating that survivin expression was affected by the Pgp inhibitor verapamil. In this paper, approximately $90 \%$ of the survivin expression was retarded after incubation with verapamil for 12 $\mathrm{h}$ in MCF-7/ADR cells (Figs. 2A, C). This finding implies that survivin correlates with Pgp in the development of MDR directly or indirectly. Survivin is a protein which travels frequently between the cytoplasm and nuclear locations and it requires an active temperature-sensitive import mechanism. Growing evidence has indicated that there is mitochondrial localization of survivin in cancer cells, ${ }^{28,29)}$ and the intracellular transport of survivin is very important for the antiapoptosis effects of the protein. ${ }^{29,30)}$ Taking all these together, the overexpression of Pgp may mediate intracellular protein transport, which might facilitate the transport of survivin from one location to another and induce hyperfunctioning of the protein not only in anti-apoptosis but also in chemoresistance.

Although there was no obvious change in Pgp mRNA expression after survivin was suppressed by its targeting RNAi plasmid psh1/survivin (Figs. 2B, D), we studied whether the change in survivin expression affects the function of Pgp (Fig. 3). Down regulation of survivin by transfection with psh1/survivin could negatively affect the function of Pgp, whilst up regulation of survivin by transfection with $\mathrm{pEGFP/}$ 
survivin could induce exclusion of the drug by cells. Modulation of survivin can affect the stability and activity of the drug transporter Pgp, suggesting it is a potential pathway through which we can control drug resistance mediated by Pgp.

Slight sensitization to ADR and MIT was detected in MCF-7 transfected with pEGFP/survivin, however, the sensitization to ADR was less than that to MIT (Table 1). As ADR was the substrate of Pgp, this result demonstrates that survivin could influence the function of Pgp.

In summary, the present results demonstrate that survivin plays a role in the chemoresistance mediated by Pgp in cancer cells. Survivin might modulate the turnover of Pgp or transport by Pgp in the cell, which then results in anti-apoptosis and drug resistance. We have only studied the change in mRNA expression between survivin and Pgp. Further studies should concentrate on protein expression using knocking down systems to clarify the mechanism responsible for survivin as well as Pgp in multidrug resistance. Notwithstanding its limitation, this study does suggest that survivin plays a role in MDR in the presence of Pgp. This might represent a novel strategy for modulating MDR in cancer cells.

Acknowledgements This work was supported by the National Natural Science Foundation of China (Grant No. 20572060), the Key Fundamental Research Foundation of China (Grant No. 2005CCAO3400), and the Department of Science and Technology of Guangdong Province (Grant No. 2005A11601008).

\section{REFERENCES}

1) Ross D. D., Leukemia, 14, 467-473 (2000).

2) Roninson I. B., Biochem. Pharmacol., 43, 95-102 (1992).

3) Shabbits J. A., Mayer L. D., Mol. Cancer Ther, 1, 205-213 (2002).

4) Ambrosini G., Adida C., Altieri D. C., Nat. Med., 3, 917-921 (1997).

5) Li F., Ambrosini G., Chu E. Y., Plescia J., Tognin S., Marchisio P. C., Altieri D. C., Nature (London), 396, 580-584 (1998).

6) LaCasse E. C., Baird S., Korneluk R. G., MacKenzie A. E., Oncogene, 17, 3247-3259 (1998).

7) Tamm I., Wang Y., Sausville E., Scudiero D. A., Vigna N., Oltersdorf
T., Reed J. C., Cancer Res., 58, 5315-5320 (1998).

8) Deveraux Q. L., Reed J. C., Genes Dev., 13, 239-252 (1999).

9) Shin S., Sung B. J., Cho Y. S., Kim H. J., Ha N. C., Hwang J. I., Chung C. W., Jung Y. K., Oh B. H., Biochemistry, 40, 1117-1123 (2001).

10) O’Connor D. S., Grossman D., Plescia J., Li F., Zhang H., Villa A., Tognin S., Marchisio P. C., Altieri D. C., Proc. Natl. Acad. Sci. U.S.A., 97, 13103-13107 (2000).

11) Song Z., Yao X., Wu M., J. Biol. Chem., 278, 23130-23140 (2003).

12) Altieri D. C., J. Cell Biochem., 92, 656-663 (2004).

13) Zhang M., Latham D. E., Delaney M. A., Chakravarti A., Oncogene, 24, 2474-2482 (2005).

14) Tran J., Master Z., Yu J. L., Rak J., Dumont D. J., Kerbel R. S., Proc. Natl. Acad. Sci. U.S.A., 99, 4349-4354 (2002).

15) Rodel C., Haas J., Groth A., Grabenbauer G. G., Sauer R., Rodel F., Int. J. Radiat. Oncol. Biol. Phys., 55, 1341-1347 (2003).

16) Wang Z. H., Xie Y. H., Wang H. B., Cancer Biol. Ther., 4, 716-719 (2005).

17) Ikeguchi M., Liu J., Kaibara N., Apoptosis, 7, 23-29 (2002).

18) Cohen J. S., Lyon R. C., Chen C., Faustino P. J. Batist G., Shoemaker M., Rubalcaba E., Cowan K. H., Cancer Res., 46, $4087-4090$ (1986).

19) Ambudkar S. V., Kimchi-Sarfaty C., Sauna Z. E., Gottesman M. M., Oncogene, 22, 7468-7485 (2003).

20) Nooter K., Herweijer H., Br. J. Cancer, 63, 663-669 (1991).

21) Adida C., Berrebi D., Peuchmaur M., Reyes-Mugica M., Altieri D. C., Lancet, 351, 882 -883 (1998).

22) Monzo M., Rosell R., Felip E., Astudillo J., Sanchez J. J., Maestre J., Martin C., Font A., Barnadas A., Abad A., J. Clin. Oncol., 17, 2100 2104 (1999).

23) Tirro E., Consoli M. L., Massimino M., Manzella L., Frasca F., Sciacca L., Vicari L., Stassi G., Messina L., Messina A., Vigneri P., Cancer Res., 66, 4263-4272 (2006).

24) Notarbartolo M., Cervello M., Dusonchet L., Cusimano A., D’Alessandro N., Cancer Lett., 180, 91-101 (2002).

25) Nakagawa Y., Abe S., Kurata M., Hasegawa M., Yamamoto K., Inoue M., Takemura T., Suzuki K., Kitagawa M., Am. J. Hematol., 81, 824831 (2006).

26) Cornwell M. M., Pastan I., Gottesman M. M., J. Biol. Chem., 262, 2166-2170 (1987).

27) Muller C., Goubin F., Ferrandis E., Mol. Pharmacol., 47, 51-56 (1995).

28) Ling X., Yang J., Tan D. F., Ramnath N., Younis T., Bundy B. N., Slocum H. K., Yang L. L., Zhou M. X., Li F. Z., Lung Cancer, 49, 353-361 (2005)

29) Dohi T., Beltrami E., Wall N. R., Plescia J., Altieri D. C., J. Clin. Invest., 114, 1117-1127 (2004).

30) Rodriguez J. A., Span S. W., Ferreira C. G. M., Kruyt F. A. E., Giaccone G., Exp. Cell Res., 275, 44-53 (2002). 